\title{
Tibial or hip BMD predict clinical fracture risk equally well: results from a prospective study in $\mathbf{7 0 0}$ elderly Swiss women
}

\author{
A. W. Popp • C. Senn • O. Franta • M. A. Krieg • \\ R. Perrelet $\cdot$ K. Lippuner
}

Received: 7 August 2008 / Accepted: 7 November 2008 / Published online: 19 December 2008

(C) International Osteoporosis Foundation and National Osteoporosis Foundation 2008

\begin{abstract}
Summary In a randomly selected cohort of Swiss communitydwelling elderly women prospectively followed up for $2.8 \pm$ 0.6 years, clinical fractures were assessed twice yearly. Bone mineral density (BMD) measured at tibial diaphysis (T-DIA) and tibial epiphysis (T-EPI) using dual-energy X-ray absorptiometry (DXA) was shown to be a valid alternative to lumbar spine or hip BMD in predicting fractures.

Introduction A study was carried out to determine whether BMD measurement at the distal tibia sites of T-EPI and TDIA is predictive of clinical fracture risk.

Methods In a predefined representative cohort of Swiss community-dwelling elderly women aged 70-80 years included in the prospective, multi-centre Swiss Evaluation of the Methods of Measurement of Osteoporotic Fracture risk (SEMOF) study, fracture risk profile was assessed and BMD measured at the lumbar spine (LS), hip (HIP) and tibia (T-DIA and T-EPI) using DXA. Thereafter, clinical fractures were reported in a bi-yearly questionnaire.

Results During 1,786 women-years of follow-up, 68 clinical fragility fractures occurred in 61 women. Older age and previous fracture were identified as risk factors for the present fractures. A decrease of 1 standard deviation in BMD values yielded a 1.5 -fold (HIP) to 1.8 -fold (T-EPI) significant increase in clinical fragility fracture hazard ratio
\end{abstract}

\footnotetext{
A. W. Popp $\cdot$ C. Senn $\cdot$ O. Franta $\cdot$ R. Perrelet $\cdot$ K. Lippuner $(\bowtie)$

Osteoporosis Policlinic, Inselspital,

Bern University Hospital and University of Bern,

CH-3010 Bern, Switzerland

e-mail: kurt.lippuner@insel.ch

M. A. Krieg

Unit of Bone Diseases, Fribourg Hospital,

Riaz, Switzerland
}

(adjusted for age and previous fracture). All measured sites had comparable performance for fracture prediction (area under the curve range from 0.63 [LS] to 0.68 [T-EPI]). Conclusion Fracture risk prediction with BMD measurements at T-DIA and T-EPI is a valid alternative to BMD measurements at LS or HIP for patients in whom these sites cannot be accessed for clinical, technical or practical reasons.

Keywords Bone densitometry - Distal tibia .

Elderly women · Fracture risk prediction · Osteoporosis

\section{Introduction}

Bone mineral density (BMD) measurements by dual-energy X-ray absorptiometry (DXA) are established worldwide as the standard examination for identification of patients with low bone mass and for monitoring BMD changes during therapy with bone active substances. Low BMD values are predictive of individual fracture risk. However, whilst this has been clearly established for central measurement sites of the skeleton, i.e. hip, lumbar spine and one third radius, to some extent [1], less is known with regard to other peripheral measurement sites, such as the tibia.

The tibia is a weight-bearing bone, easily accessible for peripheral BMD measurements of its distal third. Peripheral BMD measurements are of particular interest in elderly patients in whom artefacts, e.g. due to osteoarthritis, may preempt the diagnosis of low bone mass from the central skeletal sites, and even more so if the stringent criteria of BMD determination of the International Society for Clinical Densitometry (ISCD) are applied, such as the sequential exclusion of anatomically abnormal vertebrae [1]. They are also of interest in patients with metabolic bone diseases or 
with specific forms of osteoporosis affecting the weightbearing bones, such as immobilisation osteoporosis in paraplegics.

Whereas the tibial epiphysis (T-EPI) predominantly consists of trabecular bone, tibial diaphysis (T-DIA) predominantly contains cortical bone. Earlier research in postmenopausal women and in women with primary hyperparathyroidism showed that BMD measurements at T-EPI and T-DIA were suitable for assessing bone loss and bone mass distribution between the trabecular and cortical compartments and for discriminating between the two with high precision and reproducibility [2]. Furthermore, in patients with immobilisation osteoporosis after spinal cord injury (SCI), BMD was shown to decrease over time at infralesional measurement sites including the tibia but not at supralesional sites, such as the distal forearm [3]. In addition, tibial bone loss after SCI was shown to be significantly reduced by alendronate treatment over 2 years, at both T-EPI and T-DIA, indicating that treatment effects might be monitored by regular BMD measurement at the distal third of the tibia [4]. Whether BMD measured at TEPI and T-DIA is predictive of fracture risk remains unknown.

The aim of the present study was to determine whether BMD measurement at the distal tibia sites of T-EPI and TDIA is predictive of clinical fracture risk.

\section{Materials and methods}

Population

In a predefined cohort of elderly (70 to 80 years old) women who were included in the previously published prospective, multi-centre Swiss Evaluation of the Methods of Measurement of Osteoporotic Fracture risk (SEMOF) study [5], BMD was measured using DXA. Women were randomly recruited from official state registries between January 1998 and April 2000 and were included in SEMOF if able to walk and being independent for their daily activities. Women with a history of hip fracture or of bilateral hip replacement were not admitted. Those who decided to attend their visit at the SEMOF site of the Osteoporosis Policlinic of the University Hospital of Bern, Switzerland were included in the present study.

Questionnaires and BMD measurements

At baseline, patient history was collected in a face-to-face interview, including personal history of non-vertebral fracture, maternal history of hip fracture, age at menopause, use of oestrogens and/or corticosteroids, past and present smoking and alcohol intake habits, past and present osteoporosis treatment and propensity to falls. In addition, a clinical examination was carried out. Weight was measured on a standard beam scale without shoes and heavy outer clothing and height was determined using the average of two consecutive measurements using a Harpenden ${ }^{\mathrm{TM}}$ stadiometer. Furthermore, a chair test (ability to stand up three times from a chair without using the arms) was performed to assess the participant's propensity to falls [6].

BMD was measured at the lumbar spine (LS, first to fourth lumbar vertebrae) and at the non-dominant (nonfractured) total hip (HIP), femoral neck (NECK), trochanter (TROCH), distal tibial diaphysis (T-DIA) and distal tibial epiphysis (T-EPI) using DXA (Hologic QDR 4500A ${ }^{\mathrm{TM}}$, Hologic, Bedford, MA, USA). Scans were performed according to the manufacturer's guidelines. The standardised procedure of tibial measurements was published earlier by our group [2]. Briefly, the region of interest (ROI) is defined as the area of $120 \mathrm{~mm}$ height and $129 \mathrm{~mm}$ width, starting $10 \mathrm{~mm}$ above the top of the ankle joint space. TEPI corresponds to the distal $40 \mathrm{~mm}$ of the ROI and T-DIA to the proximal $40 \mathrm{~mm}$ of this ROI. For repeated longitudinal measurements, mean precision error of this method was $1.4 \%$ and $2.1 \%$ for T-EPI and T-DIA, respectively [2].

BMD was expressed as grams per square centimetre of hydroxyapatite and as $T$-scores (standard deviation [SD] from the mean of a healthy young female population). Whereas the National Health and Nutrition Examination Survey III (NHANES III) database [7-9] served as reference for all hip sites, the manufacturer's normative database was used as reference for the lumbar spine after analysis according to ISCD rules [1]. Thus, all evaluable vertebrae from L1 to L4 were used. Vertebrae that were affected by local structural changes or artefacts were excluded from analysis if anatomically clearly abnormal and non-assessable within the resolution of the system or if the $T$-score difference with the adjacent vertebrae exceeded 1.0 SD. When vertebrae were excluded, BMD of the remaining vertebrae was used to derive the $T$-score. Three vertebrae were used if four could not, two were used if three could not. Since BMD-based diagnostic classification should not be made on a single vertebra, the lumbar spine scan was judged non-evaluable in these cases.

For tibial BMD, the local Bern normative database derived from 400 healthy Caucasian women living in the area of Bern, Switzerland served as reference [10]. Peak bone mass (mean value \pm SD of the age group 20 to 29 years) in this female reference population is $1.278 \pm 0.116 \mathrm{~g} / \mathrm{cm}^{2}$ (T-DIA), and $0.763 \pm 0.094 \mathrm{~g} / \mathrm{cm}^{2}$ (T-EPI), respectively.

Quality control was performed daily (anthropometric spine phantom supplied by the manufacturer) with an overall precision error of $0.3 \%$ in vitro and a mean precision error in our hands of $1.1 \%$ in vivo (LS). 
After the first and only mandatory visit, each patient agreed returning a questionnaire every 6 months by mail, registering any health changes, including any fracture that occurred during the time interval between two questionnaires. For every fracture, the exact localisation and trauma intensity were to be indicated. Low trauma or fragility fractures were defined as either spontaneous or consecutive to a fall from standing height or less. Each reported clinical fracture was confirmed by a specific questionnaire addressed either to the family practitioner or to the hospital in charge of the participant, as requested by the patient. Follow-up was completed in October 2002.

\section{Statistics}

After checking for normal distribution, results of all parameters were expressed as means and SD. Simple regression analysis was applied to determine intersite correlations between BMD at the various sites of measurement.

The hazard of the first clinical fracture was calculated by using the proportional hazards model of Cox. For women who experienced one or several clinical fractures, the timespan between date of enrolment and date of the fracture event was recorded. Hazard ratios (HR) are reported with their corresponding $95 \%$ confidence intervals $(95 \% \mathrm{CI})$. They express the effect of a reduction of $1 \mathrm{SD}$ in BMD at each site, adjusted for significant predictors of clinical fracture risk in this cohort, i.e. age $(p=0.002)$ and previous fracture $(p=0.03)$ determined by Cox regression analysis. The predictive value for clinical fracture risk of each measurement site was determined by calculating the area under the receiver operating characteristic (ROC) curve using logistic regression. The differences between the areas under the curves (AUCs) were tested by using the method of DeLong et al. [11]. All analyses were performed with a Stata 8.1 statistical software package (Stata, College Station, TX, USA).

The primary goal of the SEMOF study was to evaluate the potential of quantitative ultrasound devices for fracture risk prediction. This study was mandated by the Federal Office of Social Insurances and financed by the Association of Swiss Health Insurances. A random sample of 56,561 Swiss women aged 70 to 80 years, generated by an independent state office for addresses, the "Bureau vaudois d'adresses", was contacted by mail. In this sample, 7,562 women agreed being included in the study in one of the ten participating osteoporosis centres, usually choosing the one closest to their place of living. The sub-study with tibial and other BMD measurements was initiated by the local investigator from the Bern SEMOF site and sponsored by the independent scientific fund of the Osteoporosis Policlinic of the University Hospital and University of Bern. The study protocol was accepted by the Swiss ethics committee for medical sciences. All women gave their written informed consent before inclusion.

\section{Results}

Seven hundred and one randomly selected women from the region of Bern, $76.1 \pm 3.0$ years old in average and with mean BMI $25.8 \pm 4.1 \mathrm{~kg} / \mathrm{m}^{2}$, were included in the study at the SEMOF site of Bern. Of these, 14 women had no baseline DXA measurement and another 50 (7.1\%) were lost for follow-up (i.e. did not return the first questionnaire due 6 months after the inclusion visit). Drop-outs did not differ significantly from participants remaining in the study with regard to age, BMI and BMD at the various sites.

The remaining 637 women who were included in the present study were $(m e a n \pm S D) 76.0 \pm 3.0$ years old with a BMI of $25.8 \pm 4.3 \mathrm{~kg} / \mathrm{m}^{2}$. The participants were followed up during a mean observation period of $2.8 \pm 0.6$ years (range 0.12 to 3.94 years), corresponding to a global follow-up of 1,786 women-years. During this follow-up, 68 clinical fragility fractures occurred in 61 women. The fracture sites were: forearm $(n=25)$, vertebrae $(n=13)$, proximal humerus $(n=12)$, hip and pelvis $(n=8)$, ankle $(n=4)$, elbow $(n=2)$, clavicle $(n=1)$, rib $(n=1)$, patella $(n=1)$ and tibia $(n=1)$.

Baseline demographics and detailed BMD values are shown in Table 1. Compared to participants who did not present a clinical fracture during the duration of the observation, those who did were significantly older (76.9 vs. 75.9 years, $p=0.02$ ) and had more often a personal history of fracture at baseline ( $66 \%$ vs. $51 \%, p=0.03)$. However, clinical fracture patients did not significantly differ from the remaining women with respect to maternal history of hip fracture $(6.8 \%$ vs. $11 \%, p=$ n.s. $)$, current use of oestrogens ( $5 \%$ vs. $12 \%, p=$ n.s.), current or past use of corticosteroids ( $8 \%$ vs. $3.7 \%, p=$ n.s.), smoking $(4.9 \%$ vs. $5.8 \%, p=$ n.s.), alcohol intake ( $31.5 \%$ vs. $39.7 \%, p=$ n.s.), history of falls (one or more falls during the preceding year, $34 \%$ vs. $26 \%, p=$ n.s.), current or past osteoporosis therapy (5\% vs. $2.3 \%, p=$ n.s.) and feasibility of the chair test (96.7\% vs. $97.2 \%, p=$ n.s.).

Whereas all tibia and hip scans were evaluable, ISCD rules led to the exclusion of $68 \mathrm{LS}$ scans (i.e. $>10 \%$ of all LS scans). Participants with an incident clinical fracture had significantly lower BMD at all measured sites compared to those without (Table 1). The largest differences in mean $T$ scores between the two groups were found at the LS (after analysis according to ISCD, which reduced the number of evaluable LS scans in the fracture group from 61 to 56) and at both tibial sites. Fifty-three percent (30/56) of the women with incident fractures had an initial $T$-score of $-2.5 \mathrm{SD}$ or lower at the LS, 41\% (25/61) at the NECK and 46\% (28/ $61)$ at the T-EPI, respectively. The respective specificities 
Table 1 Demographic and BMD values at various sites of the 637 women who were available for follow-up examination (mean \pm SD)

\begin{tabular}{|c|c|c|c|c|}
\hline Parameter & All women $(n=637)$ & No clinical fracture $(n=576)$ & Clinical fracture $(n=61)$ & $p$ value \\
\hline Age (years) & $76.0(3.0)$ & $75.9(3.0)$ & $76.9(2.8)$ & 0.02 \\
\hline Age at menopause (years) & $49.1(4.5)$ & $49.1(4.5)$ & $48.3(4.0)$ & 0.2 (n.s.) \\
\hline Weight $(\mathrm{kg})$ & $64.7(10.9)$ & $64.8(10.9)$ & $63.0(11.1)$ & 0.2 (n.s.) \\
\hline BMI $\left(\mathrm{kg} / \mathrm{m}^{2}\right)$ & $25.8(4.3)$ & $25.9(4.2)$ & $25.3(4.5)$ & 0.3 (n.s.) \\
\hline Previous fracture $(\%)$ & 52 & 51 & 66 & 0.03 \\
\hline Follow-up (days) & $1,024(233)$ & $1,029(227)$ & $983(275)$ & 0.2 (n.s.) \\
\hline \multicolumn{5}{|l|}{ LS } \\
\hline BMD L1-L4 (g/cm²) & $0.898(0.178)$ & $0.906(0.178)^{\mathrm{a}}$ & $0.821(0.170)$ & 0.0004 \\
\hline BMD L2-L4 (g/cm²) & $0.936(0.007)$ & $0.944(0.187)^{\mathrm{b}}$ & $0.858(0.174)$ & 0.0006 \\
\hline$T$-score ISCD (SD) & $-1.82(1.40)$ & $-1.74(1.38)^{\mathrm{c}}$ & $-2.53(1.37)^{\mathrm{d}}$ & $<0.0001$ \\
\hline \multicolumn{5}{|l|}{ HIP (NHANES) } \\
\hline $\operatorname{BMD}\left(\mathrm{g} / \mathrm{cm}^{2}\right)$ & $0.770(0.121)$ & $0.776(0.119)$ & $0.714(0.123)$ & 0.0001 \\
\hline$T$-score (SD) & $-1.42(1.00)$ & $-1.37(0.98)$ & $-1.88(1.01)$ & 0.0001 \\
\hline \multicolumn{5}{|l|}{ NECK } \\
\hline $\mathrm{BMD}\left(\mathrm{g} / \mathrm{cm}^{2}\right)$ & $0.653(0.105)$ & $0.658(0.104)$ & $0.600(0.107)$ & $<0.0001$ \\
\hline$T$-score (SD) & $-1.78(0.95)$ & $-1.73(0.94)$ & $-2.26(0.97)$ & $<0.0001$ \\
\hline \multicolumn{5}{|l|}{ TROCH } \\
\hline $\mathrm{BMD}\left(\mathrm{g} / \mathrm{cm}^{2}\right)$ & $0.587(0.103)$ & $0.592(0.102)$ & $0.539(0.102)$ & 0.0002 \\
\hline$T$-score (SD) & $-1.16(1.03)$ & $-1.10(1.02)$ & $-1.64(1.02)$ & 0.0001 \\
\hline \multicolumn{5}{|l|}{ T-DIA } \\
\hline $\operatorname{BMD}\left(\mathrm{g} / \mathrm{cm}^{2}\right)$ & $1.163(0.156)$ & $1.170(0.154)^{\mathrm{e}}$ & $1.093(0.158)$ & 0.0002 \\
\hline$T$-score (SD) & $-1.00(1.35)$ & $-0.93(1.33)^{\mathrm{e}}$ & $-1.63(1.37)$ & 0.0001 \\
\hline \multicolumn{5}{|l|}{ T-EPI } \\
\hline $\operatorname{BMD}\left(\mathrm{g} / \mathrm{cm}^{2}\right)$ & $0.599(0.103)$ & $0.606(0.103)^{\mathrm{e}}$ & $0.541(0.089)$ & $<0.0001$ \\
\hline$T$-score (SD) & $-1.73(1.10)$ & $-1.67(1.10)^{\mathrm{e}}$ & $-2.37(0.95)$ & $<0.0001$ \\
\hline
\end{tabular}

${ }^{\mathrm{a}} n=562$

$\mathrm{b}_{n=568}$

${ }^{\mathrm{c}} n=513$

d $n=56$

${ }^{\mathrm{e}}{ }_{n=575}$

were $69 \%$ (LS), 78\% (NECK) and 75\% (T-EPI). If all three sites were taken together, sensitivity increased to $69 \%$ (42/ 61 ) whereas specificity decreased to $55 \%$.

Table 2 shows the intersite correlations for BMD values at the various sites. The correlation coefficients ranged from 0.499 (between T-DIA and L2-L4) to 0.905 (between TROCH and HIP). The highest correlation between two distinct bones was found between T-EPI and HIP with an $r$ value of 0.716 .
As shown in Table 3, a decrease of $1 \mathrm{SD}$ in BMD Tscore value yielded a 1.5 -fold (HIP) to 1.8 -fold (T-EPI) significant increase of the clinical fragility fracture HR (adjusted for age and previous fracture). The areas under the ROC curves (AUC) were calculated in order to compare the performance for clinical fracture risk prediction between the individual measurement sites (Table 3). AUCs ranged from 0.63 (LS) to 0.68 (T-EPI) with no significant differences between the sites measured.

Table 2 Intersite correlations $(r)$ of BMD

\begin{tabular}{lclllll}
\hline Site & LS (L2-L4) & LS (L1-L4) & ISCD T-score & NECK & TROCH & HIP \\
\hline LS (L2-L4) & 1.000 & & & & & \\
LS (L1-L4) & 0.993 & 1.000 & 0.947 & 1.000 & & \\
ISCD T-score & 0.931 & 0.576 & 0.588 & 1.000 & & \\
NECK & 0.568 & 0.618 & 0.619 & 0.765 & 1.000 & 1.000 \\
TROCH & 0.608 & 0.631 & 0.640 & 0.862 & 0.905 & 0.644 \\
HIP & 0.622 & 0.510 & 0.528 & 0.509 & 0.558 & 1.000 \\
T-DIA & 0.499 & 0.588 & 0.610 & 0.608 & 0.677 & 0.716 \\
T-EPI & 0.577 & & & & \\
\hline
\end{tabular}


Table 3 Adjusted HR of clinical fractures attributed to a decrease of $1 \mathrm{SD}$ in BMD and predictive power of BMD variables for clinical fracture using the area under the ROC curves (AUC)

\begin{tabular}{|c|c|c|c|c|c|c|}
\hline BMD variables & $N$ & SE & SD & Coefficient & HR $(95 \% \mathrm{CI})$ & AUC (95\%CI) \\
\hline \multicolumn{7}{|l|}{ Lumbar spine } \\
\hline L2-L4 & 629 & 0.73 & 0.188 & 2.37 & $1.6(1.2,2.0)$ & $0.63(0.56,0.70)$ \\
\hline $\mathrm{L} 1-\mathrm{L} 4$ & 623 & 0.78 & 0.178 & 2.59 & $1.6(1.2,2.1)$ & $0.63(0.56,0.70)$ \\
\hline ISCD $T$-score & 569 & 0.10 & 1.399 & 0.39 & $1.7(1.3,2.3)$ & $0.65(0.58,0.72)$ \\
\hline \multicolumn{7}{|l|}{ Hip (Hologic-NHANES) } \\
\hline Neck & 636 & 1.24 & 0.105 & 4.82 & $1.7(1.3,2.1)$ & $0.65(0.58,0.72)$ \\
\hline Trochanter & 636 & 1.15 & 0.103 & 3.81 & $1.5(1.2,1.9)$ & $0.64(0.57,0.72)$ \\
\hline Total hip & 636 & 0.99 & 0.121 & 3.44 & $1.5(1.2,1.9)$ & $0.64(0.56,0.71)$ \\
\hline \multicolumn{7}{|l|}{ Tibia } \\
\hline Tibial diaphysis BMD & 636 & 0.82 & 0.156 & 2.74 & $1.5(1.2,2.0)$ & $0.64(0.57,0.71)$ \\
\hline Tibial epiphysis BMD & 636 & 1.35 & 0.103 & 5.67 & $1.8(1.4,2.4)$ & $0.68(0.61,0.74)$ \\
\hline
\end{tabular}

Adjusted for age and past fracture using Cox regression

\section{Discussion}

In the present predefined cohort of 701 elderly women, 687 had their BMD measured at the lumbar spine, hip, and tibial epiphysis and diaphysis. They were prospectively followed up during almost 3 years with regard to fracture events. Each decrease in BMD of $1 \mathrm{SD}$ resulted in a consistent increase in clinical fracture HR ranging between 1.5 (for tibial diaphysis, trochanter and total hip) and 1.8 (for tibial epiphysis). There was no significant difference in predictive value for clinical fractures between the different BMD measurement sites. BMD measurement at the tibia (T-EPI or T-DIA) can, therefore, be considered as being of similar predictive value than BMD measurement at the lumbar spine or the hip.

These findings are consistent with previously published observations. In the meta-analysis of Marshall et al. [12], including 11 studies with more than 90,000 person-years of observation and more than 2,000 fractures, all measured sites (limited to the spine, hip and radius) had similar predictive ability for global fracture risk with a relative risk (RR) of 1.4-1.6 per SD loss of BMD with the exceptions of LS measurements for predicting vertebral fractures $(R R=$ 2.3) and of hip measurements for predicting hip fractures $(\mathrm{RR}=2.6)$. Interestingly, the predictive value of a $1 \mathrm{SD}$ decrease in BMD was shown to be comparable to that of a $1 \mathrm{SD}$ increase in blood pressure for stroke and better than a 1 SD increase in serum cholesterol for cardiovascular disease [12]. The National Osteoporosis Risk Assessment (NORA) study reported similar results for heel, forearm and finger BMD [13]. These findings were confirmed by the 10-year data of the Study of Osteoporotic Fractures (SOF), which reported RR values around 1.4 for six different BMD measurement sites (distal and proximal radius, heel, spine, total hip and femoral neck) [14]. In the present study, the adjusted HR for any clinical fracture attributed to a decrease of $1 \mathrm{SD}$ in BMD was 1.5 for T-DIA and 1.8 for T-EPI.

Tibial BMD measurement has been shown to be useful in the context of immobilisation osteoporosis after spinal cord injury [3-4]. However, it may also become important in a variety of other clinical situations. In patients with severe osteoarthritis of the spine or hip and in patients who underwent hip or spine surgery, e.g. hip replacement or scoliosis fixation/vertebroplasty, and have orthopaedic implants, BMD must be measured at a peripheral site. Furthermore, in the present study, the ISCD criteria applied to lumbar spine BMD analysis resulted in a loss of almost $10 \%$ of all evaluable patients. There also, BMD measurement at the tibia may be a suitable alternative. In patients with metabolic bone diseases, such as hyperparathyroidism, cortical bone loss is known to be more pronounced than trabecular bone loss [15-16] and these patients are at increased risk for fractures [17]. In dialysed renal insufficiency patients, earlier research has provided evidence for the preservation of cortical bone (measured at T-DIA) with continuous ambulatory peritoneal dialysis compared to haemodialysis, possibly in relationship with the higher residual renal function observed in the former [18]. Furthermore, in the very specific population of female to male transsexuals under long-term hormonal treatment, the effect of androgens on cortical bone was shown in a crosssectional study measuring BMD at the tibial sites [10]. In idiopathic renal stone formers, BMD was previously shown to be reduced at LS, Ward's triangle, T-EPI and T-DIA, whereby the latter two showed the largest magnitudes of change [19]. Although the mechanism behind this possible site selectivity remains unknown, this observation documents that tibial measurements may give access to not yet understood pathophysiological mechanisms in specific patient populations and/or metabolic bone diseases. 
In the tibia, cortical (T-DIA) and predominantly trabecular (T-EPI) bone regions can be measured within the same weight-bearing bone, thereby reducing the potential for differences in BMD related to different localisations in the body. BMD measurement at the tibia does not only reflect the distribution of bone loss in the two compartments [2], but should now also be considered as predictive for clinical fracture risk. Paraplegics are at increased fracture risk due to the massive and sustained bone loss they experience in their lower limbs. In paraplegic men, BMD at T-EPI and TDIA was shown to follow a different decrease pattern, tibial epiphysis showing a rapid demineralisation which levels off 3 years after SCI and comparable to observations made at the femoral neck level and tibial diaphysis showing a slower but continuous demineralisation which proceeds even beyond 10 years post-injury. In contrast, no such bone loss was observed at the supralesional BMD measurement sites [3]. Based on the results of the present study and others [4], BMD changes at the tibia may be considered as predictive for fracture risk in this patient population.

According to the World Health Organisation and ISCD guidelines, the diagnosis of osteoporosis should ideally be made on the basis of a BMD measurement performed at the femoral neck [1]. However, there are clinical situations in which these recommended measurements are not practicable, such as in patients after bilateral hip replacement (who were excluded from the present study), patients with severe osteophytosis due to hip osteoarthritis and patients with restricted hip mobility due to severe pain. Although such patients were excluded by protocol from the present study, they represent a non-negligible and even reasonably sized patient population in daily practice. For these patients, an easily accessible peripheral measurement site with good predictive value for overall clinical fracture risk is of expected clinical interest in daily practice. In the present study, the intersite correlations between BMD measured at the femoral neck and T-DIA/T-EPI were 0.509/0.608, respectively. These values were comparable to the correlation coefficient observed between lumbar spine and femoral neck. For all patients mentioned above, a BMD measurement at T-DIA and T-EPI may, therefore, become a valuable alternative or addition to a BMD measurement at the lumbar spine or femoral neck for determining their individual fracture risk. This may become especially important in women aged 65 years and older, since the increase in fracture risk prediction achieved by combining BMD measurements at the lumbar spine and femoral neck was shown to vanish due to degenerative changes of the lumbar spine [20].

Among all risk factors for fracture assessed in the present study, only age and personal history of fracture were significantly different between fractured and nonfractured patients. This contrasts with recent findings, showing that risk factors such as smoking status, maternal history of hip fracture and corticosteroid intake were significant predictors of increased fracture risk [21]. However, these recent findings rely on a meta-analysis of nine primary cohorts including more than 46,000 patients and corresponding to almost 190,000 person-years of observation to be compared with the modest 701 persons during 1,786 women-years in the present study.

This study has several strengths: it was performed longitudinally and prospectively in a random sample of the elderly Swiss female population over a prolonged period of time (almost 3 years) and had a very low dropout rate (less than $8 \%$ ). It does, however, have some limitations. The low number of fractures precluded an evaluation of the HR and AUCs by fracture type. Only clinical fractures were reported, whether the same conclusions would apply to morphometric vertebral fractures remains unknown. Whether T-EPI would be more appropriate to predict fracture risk at sites with high trabecular bone contents (such as the trochanter or the spine) and whether T-DIA would be more appropriate for fracture risk at predominantly cortical sites could not be determined. Furthermore, areas of future research should include the study of the value of T-DIA and T-EPI BMD measurements for treatment monitoring in patient populations where changes in BMD are expected to be less dramatic than in paraplegics, such as postmenopausal women treated with bone active substances, and the study of the added value of tibial BMD measurements combined to other measurement sites, such as LS and HIP, in elderly patients.

In conclusion, fracture risk prediction with BMD measurements at the peripheral tibial sites T-DIA and T-EPI is a valid alternative to BMD measurements at the lumbar spine and the hip for patients in whom these sites cannot be accessed for clinical, technical or practical reasons.

Acknowledgements We are grateful to Dr. Philippe Kress for his invaluable contribution to the manuscript.

Conflicts of interest None.

\section{References}

1. International Society for Clinical Densitometry (ISCD) official positions 2007. Available at http://www.iscd.org/Visitors/positions/ OfficialPositionsText.cfm. Accessed October 24, 2008

2. Casez JP, Troendle A, Lippuner K, Jaeger P (1994) Bone mineral density at distal tibia using dual-energy X-ray absorptiometry in normal women and in patients with vertebral osteoporosis or primary hyperparathyroidism. J Bone Miner Res 9(12):1851-1857

3. Zehnder Y, Lüthi M, Michel D et al (2004) Long-term changes in bone metabolism, bone mineral density, quantitative ultrasound parameters, and fracture incidence after spinal cord injury: a cross- 
sectional observational study in 100 paraplegic men. Osteoporos Int 15(3):180-189

4. Zehnder Y, Risi S, Michel D et al (2004) Prevention of bone loss in paraplegics over 2 years with alendronate. J Bone Miner Res 19 (7):1067-1074

5. Krieg MA, Cornuz J, Ruffieux C (2003) Comparison of three bone ultrasounds for the discrimination of subjects with and without osteoporotic fractures among 7562 elderly women. J Bone Miner Res 18(7):1261-1266

6. Terwee CB, Mokkink LB, Steultjens MP, Dekker J (2006) Performance-based methods for measuring the physical function of patients with osteoarthritis of the hip or knee: a systematic review of measurement properties. Rheumatology (Oxford) 45(7):890-902

7. Looker AC, Wahner HW, Dunn WL et al (1995) Proximal femur bone mineral levels of US adults. Osteoporos Int 5(5):389-409

8. Looker AC, Orwoll ES, Johnston CC Jr et al (1997) Prevalence of low femoral bone density in older U.S. adults from NHANES III. J Bone Miner Res 12(11):1761-1768

9. Looker AC, Wahner HW, Dunn WL et al (1998) Updated data on proximal femur bone mineral levels of US adults. Osteoporos Int 8(5):468-489

10. Ruetsche AG, Kneubuehl R, Birkhaeuser MH, Lippuner K (2005) Cortical and trabecular bone mineral density in transsexuals after long-term cross-sex hormonal treatment: a cross-sectional study. Osteoporos Int 16(7):791-798

11. DeLong ER, DeLong DM, Clarke-Pearson DL (1988) Comparing the areas under two or more correlated receiver operating characteristic curves: a nonparametric approach. Biometrics 44(3):837-845

12. Marshall D, Johnell O, Wedel H (1996) Meta-analysis of how well measures of bone mineral density predict occurrence of osteoporotic fractures. BMJ 312(7041):1254-1259
13. Miller PD, Siris ES, Barrett-Connor E et al (2002) Prediction of fracture risk in postmenopausal white women with peripheral bone densitometry: evidence from the National Osteoporosis Risk Assessment. J Bone Miner Res 17(12):2222-2230

14. Stone KL, Seeley DG, Lui LY et al (2003) BMD at multiple sites and risk of fracture of multiple types: long-term results from the Study of Osteoporotic Fractures. J Bone Miner Res 18(11):19471954

15. Seeman E, Wahner HW, Offord KP, Kumar R, Johnson WJ, Riggs BL (1982) Differential effects of endocrine dysfunction on the axial and the appendicular skeleton. J Clin Invest 69(6):13021309

16. Silverberg SJ, Shane E, de la Cruz L et al (1989) Skeletal disease in primary hyperparathyroidism. J Bone Miner Res 4(3):283-291

17. Khosla S, Melton J 3rd (2002) Fracture risk in primary hyperparathyroidism. J Bone Miner Res 17(Suppl 2):N103-N107

18. Mottet JJ, Horber FF, Casez JP, Descoeudres C, Jaeger P (1996) Evidence for preservation of cortical bone mineral density in patients on continuous ambulatory peritoneal dialysis. J Bone Miner Res 11(1):96-104

19. Jaeger P, Lippuner K, Casez JP, Hess B, Ackermann D, Hug C (1994) Low bone mass in idiopathic renal stone formers: magnitude and significance. J Bone Miner Res 9(10):1525-1532

20. Leslie WD, Lix LM, Tsang JF, Caetano PA, Manitoba Bone Density Program (2007) Single-site vs multisite bone density measurement for fracture prediction. Arch Intern Med 167 (15):1641-1647

21. Kanis JA, Oden A, Johnell O et al (2007) The use of clinical risk factors enhances the performance of BMD in the prediction of hip and osteoporotic fractures in men and women. Osteoporos Int 18 (8):1033-1046 